\title{
Overview of the Consortium for the Advanced Simulation of Light Water Reactors (CASL)
}

\author{
Joel A. Kulesza ${ }^{1, a}$, Fausto Franceschini ${ }^{1}$, Thomas M. Evans ${ }^{2}$, and Jess C. Gehin ${ }^{2}$ \\ ${ }^{1}$ Westinghouse Electric Company LLC 1000 Westinghouse Drive, Cranberry Township, PA 16066, \\ USA \\ ${ }^{2}$ Oak Ridge National Laboratory P.O. Box 2008 Oak Ridge, TN 37831-6003, USA
}

\begin{abstract}
The Consortium for Advanced Simulation of Light Water Reactors (CASL) was established in July 2010 for the purpose of providing advanced modeling and simulation solutions for commercial nuclear reactors. The primary goal is to provide coupled, higherfidelity, usable modeling and simulation capabilities than are currently available. These are needed to address light water reactor (LWR) operational and safety performance-defining phenomena that are not yet able to be fully modeled taking a first-principles approach. In order to pursue these goals, CASL has participation from laboratory, academic, and industry partners. These partners are pursuing the solution of ten major "Challenge Problems" in order to advance the state-of-the-art in reactor design and analysis to permit power uprates, higher burnup, life extension, and increased safety. At present, the problems being addressed by CASL are primarily reactor physics-oriented; however, this paper is intended to introduce CASL to the reactor dosimetry community because of the importance of reactor physics modelling and nuclear data to define the source term for that community and the applicability and extensibility of the transport methods being developed.
\end{abstract}

\section{Introduction}

The Consortium for Advanced Simulation of Light Water Reactors (CASL) was established in July 2010 as a U.S. Department of Energy (DOE) Energy Innovation Hub. The program was initially established as a $\$ 125 \mathrm{M}$ (USD) program with a yearly funding level of $\$ 25 \mathrm{M}$ for 5 years, with the possibility of a 5 year extension. The concept of the "Innovation Hub" derives from previous major technology initiatives that combine expertise across fields and industries such as the Manhattan Project in the 1940s and Bell Labs in the 1950s and 1960s. CASL's vision is to predict the performance of nuclear reactors through comprehensive modeling and simulation technology, based on first-principles physics, which can be used throughout the commercial nuclear energy industry to enhance safety, reliability, and economics.

The CASL team is a consortium that consists of ten core partners and numerous contributing members. The CASL organization is led by Oak Ridge National Lab (ORNL) and CASL's research and development activities are executed in six technical teams called Focus Areas (FA). This partnership provides unparalleled collective institutional knowledge, nuclear science and engineering talent, computational science leadership, and a strong record of LWR design and regulatory accomplishments.

\footnotetext{
${ }^{a}$ Corresponding author: kuleszj@westinghouse.com
} 
The main technology that drives CASL's modeling and simulations is the Virtual Environment for Reactor Applications (VERA), which provides higher-fidelity results than the current industry approach by incorporating coupled physics and science-based models, state-of-the-art numerical methods, modern computational science and software development practices, integrated uncertainty quantification (UQ) and validation against data from operating pressurized water reactors (PWRs), single-effect experiments, and integral tests. VERA integrates existing codes and methods (e.g., SCALE and Denovo [1,2]) as well as the new methods developed through CASL.

This paper will introduce the international reactor dosimetry community to CASL by describing the program's goals including the relevance of those goals to the reactor dosimetry community, the technical focus areas and core participants working in those focus areas, and the tools and resources used to solve particular "challenge problems" in those focus areas. As CASL continues, additional papers will be produced that describe the program's progress and technical results. Because of the new methodologies and tools, there will be ample opportunity to perform and report upon new methodology development and the corresponding benchmarking efforts. To follow the current state of the program, the CASL website [3] is a key resource which includes project summary slides [7] that make a particularly good and succinct resource.

\section{Goals}

\subsection{Addressing Contemporary Challenges for LWRs}

For nuclear energy to be a significant part of the electrical energy generation mix, it must be economically attractive, assuredly safe, and address high level waste management. These three characteristics are also required for public support. To ensure that the goals of CASL are in keeping with addressing these requirements, CASL has defined and is working toward solving "Challenge Problems" to address issues that are important to operating PWRs in the areas of fuel performance during normal operation, accident conditions, and plant lifetime extension.

CASL's goal is to not only provide the modeling and simulation capability to solve the specificallydefined Challenge Problems, but, by using a science-based approach, to also provide the capability to address other challenges either currently known or yet to be revealed that affect LWRs including boiling water reactors and small modular reactors having an integral PWR design. The Challenge Problems being addressed by CASL including the industry areas impacted are summarized in Table 1 .

It can be observed that many of the goals are of primary interest to the reactor physics community. However, the last two challenge problems (Reactor Vessel \& Internals Integrity) are of direct concern to the reactor dosimetry community. Because of the multi-scale and multi-physics approach taken by CASL in VERA, it is expected that improved characterization of reactor vessel behavior under operational conditions will result. For example, instead of applying iron atom displacement response functions to flux solutions, the microscopic behavior of the material can be directly simulated. Furthermore, as in-core phenomena are better modeled and simulated, the opportunities for successful analysis of retrospective dosimetry samples from in-vessel and in-core components increase. As noted previously, a better-defined source term and the transport methods developed for VERA are also expected to benefit reactor dosimetry analyses.

\subsection{Developing and Applying Modern Virtual Reactor Technology}

Currently, the traditional reactor design and analysis process relies on tools that are not fully integrated and coupled. Use of these tools typically requires a sequential analysis of different areas of physics, such as reactor neutronics, core and system thermal-hydraulics (T-H), coolant chemistry, structural analysis and fuel performance. Since the analysis tools are not fully coupled, considerable expert manpower 
Table 1. Summary of CASL Challenge Problems and Areas of Impact.

\begin{tabular}{|c|c|c|c|c|c|}
\hline & Challenge Problem & $\begin{array}{l}\text { Power } \\
\text { Uprate }\end{array}$ & $\begin{array}{l}\text { Higher } \\
\text { Burnup }\end{array}$ & $\begin{array}{c}\text { Life } \\
\text { Extension }\end{array}$ & Safety \\
\hline & CRUD-Induced & & & & \\
\hline \multirow{5}{*}{$\begin{array}{l}\text { Operational } \\
\text { Challenge } \\
\text { Problems }\end{array}$} & Power Shift & $\mathrm{X}$ & $\mathrm{X}$ & & $\mathrm{X}$ \\
\hline & $\begin{array}{c}\text { CRUD-Induced } \\
\text { Localized Corrosion }\end{array}$ & $\mathrm{X}$ & $\mathrm{X}$ & & $\mathrm{X}$ \\
\hline & $\begin{array}{l}\text { Grid-to-Rod } \\
\text { Fretting Failure }\end{array}$ & & $\mathrm{X}$ & & $\mathrm{X}$ \\
\hline & $\begin{array}{l}\text { Pellet-Clad } \\
\text { Interaction }\end{array}$ & $X$ & $X$ & & $X$ \\
\hline & $\begin{array}{l}\text { Fuel Assembly } \\
\text { Distortion }\end{array}$ & $\mathrm{X}$ & $\mathrm{X}$ & & $X$ \\
\hline \multirow{5}{*}{$\begin{array}{l}\text { Safety } \\
\text { Challenge } \\
\text { Problems }\end{array}$} & $\begin{array}{l}\text { Departure from } \\
\text { Nucleate Boiling }\end{array}$ & $\mathrm{X}$ & & & $\mathrm{X}$ \\
\hline & $\begin{array}{l}\text { Cladding Integrity during } \\
\text { Loss of Coolant Accidents }\end{array}$ & $X$ & $\mathrm{X}$ & & $X$ \\
\hline & $\begin{array}{c}\text { Cladding Integrity during } \\
\text { Reactivity Insertion Accidents }\end{array}$ & $\mathrm{X}$ & $\mathrm{X}$ & & $\mathrm{X}$ \\
\hline & $\begin{array}{c}\text { Reactor Vessel } \\
\text { Integrity }\end{array}$ & $\mathrm{X}$ & & $X$ & $X$ \\
\hline & $\begin{array}{l}\text { Reactor Internals } \\
\text { Integrity }\end{array}$ & $X$ & & $X$ & $X$ \\
\hline
\end{tabular}

is needed to make multiple conservative assumptions to ensure a conservative design, resulting in increased energy cost. A major drawback is that if downstream analyses indicate the necessity of design modifications, the process must be restarted. Frequently, much iteration is required because of the close relationships between the different technical areas involved in reactor design and analysis. The comprehensive analysis system provided by VERA allows an integrated design cycle that can more adequately account for interactions between relevant physical phenomena and reduce the need for that iteration. This system is also expected to improve analysis efficiency because of the cohesive storage and processing of input data and transfer of data between different code components (e.g., source definition, cross-section data, and radiation transport modules).

The software development methodology for VERA was planned with the understanding that software verification and validation in the commercial nuclear industry is of the utmost importance to ensure that the software is behaving correctly in all modes of operation. As such, software development undertaken by CASL uses modern software practices including rigorous verification, validation, and uncertainty quantification assessment while applying it to real-world nuclear reactor problems. Meanwhile, the software is being developed to provide more detailed analysis capabilities than what is currently available, with better mechanistic models, to support enhanced understanding that will lead to improved designs and materials. Modern software development methodologies not only enable efficiency and responsiveness to evolving requirements but also assure quality, flexibility, extensibility, reuse of software, and development efficiency. Use of these methodologies in the development of VERA demonstrates their benefits and promotes their adoption by the wider nuclear enterprise to promote better software throughout the industry. The adoption of these modern software development practices has clear benefits to the reactor dosimetry community.

\subsection{Engaging the Nuclear Energy Community through Modeling and Simulation}

From its inception, CASL was planned to engage the industrial nuclear energy community. This engagement is essential to the success and viability of the products produced by CASL. The software and analysis methodologies developed by CASL are continually exercised by the core industry partners to 
ensure that they both correctly address the challenge problems and also address the problems correctly (in a usable and effective way). As an example, since VERA contains state-of-the-art methods and technologies it can be extremely computationally intensive to the degree that it can only be run at national supercomputing laboratories. This makes it effectively unusable for typical industrial users. Therefore, throughout the development of VERA, industry feedback is continually provided to ensure that system operates in a balanced and functional fashion. This paper is also an example of that outreach: connecting with different nuclear disciplines and soliciting feedback in order to ensure that the right problems are being solved and the right people are involved in developing those solutions.

\subsection{Developing New Collaboration Paradigms}

Because of the large team of collaborators and their organizational and geographic dispersion, new collaborative technologies are needed to allow all participants to work together. In addition to advanced technology for long-distance collaboration, the organizational structure and management plans used by CASL allow all of the participants to quickly and easily collaborate with each other and to freely share information (some of which can be highly proprietary in nature).

The engagement between laboratories, academia, and industry and the collaborative platforms used in CASL ensures that a continual feedback mechanism is available to continually drive the focus on problems of interest. As CASL continues to develop, those in the reactor dosimetry community have the means and possibility to provide feedback in order to ensure that CASL (and VERA) are kept sufficiently capable to address problems that uniquely face that community. Also, as an international community, the adoption of the tools and practices proven in CASL would benefit global collaboration.

\section{Technical Focus Areas \&Core Partners}

To address the aforementioned goals and Challenge Problems, CASL research and development takes place in six inter-related focus areas contributed to by a plurality of the core partners to ensure that the right experts are working on the right problems. The focus areas are:

1. Advanced Modeling Applications (AMA),

2. Physics Integration (PHI),

3. Radiation Transport Methods (RTM),

4. Materials Performance and Optimization (MPO),

5. Validation and Uncertainty Quantification (VUQ), and

6. Thermal-Hydraulics Methods (THM).

Briefly, AMA is the primary linkage between development activities relating to existing reactors, Challenge Problems, and full-scale validation problems. AMA also dictates functional requirements, prioritizes modeling activities, and performs capability assessments.

PHI develops VERA tools by integrating the models, methods and data developed by other focus areas. PHI collaborates with AMA to deliver usable tools guided by the functional requirements set by AMA.

RTM develops next-generation neutron transport simulation tools in VERA, which consist a development path based on 3D full-core discrete ordinates $\left(\mathrm{S}_{\mathrm{N}}\right)$ transport, pin-homogenized simplified $\mathrm{P}_{\mathrm{N}}\left(\mathrm{SP}_{\mathrm{N}}\right)$ transport, and pin-resolved 2D MOC transport/1D diffusion or transport, and the advanced development path based on hybrid Monte Carlo (MC).

MPO develops improved materials performance models for fuels, cladding, and structural materials, and integrate those models into a fuel performance predictive capability, to provide better prediction of fuel and component failure and to reduce the reliance on empirical correlations. 


\section{$15^{\text {th }}$ ISRD}

VUQ provides the quantification of uncertainties and associated validation of VERA models and integrated systems where improvements in the determination of operating and safety margins will directly contribute to the ability to uprate reactors and extend their lifetimes. The methods developed by VUQ also support the transition from integral experiments to the integration of small-scale separateeffect experiments.

THM oversees the development and advancement of thermal hydraulics analysis tools and their integration with solution environments deployed on large-scale parallel computers. The primary mission of THM is to deliver code components that meet the rigorous physical model and numerical algorithm requirements of VERA.

Within these focus areas, the core laboratory partners include:

- Oak Ridge National Laboratory (ORNL),

- Idaho National Laboratory (INL),

- Los Alamos National Laboratory (LANL), and

- Sandia National Laboratories (SNL).

As noted previously, ORNL has been designated as the lead organization. The core academic partners include:

- Massachusetts Institute of Technology (MIT),

- University of Michigan (UM), and

- North Carolina State University (NCSU).

Finally, the industry partners include:

- Electric Power Research Institute (EPRI),

- Westinghouse Electric Company (WEC), and

- Tennessee Valley Authority (TVA).

There are over 20 other contributors providing expertise in all of the aforementioned focus areas.

\section{Resources}

In order to pursue the Challenge Problems and address the focus areas described previously, CASL has significant resources allocated to it. Because of the intense focus on computation, a significant allocation of computational time has been allocated on Titan (a hybrid supercomputer housed at ORNL with almost 300,000 processing cores and almost 19,000 graphics processing unit (GPU) accelerators, currently the second-fastest supercomputer in the world [4]). As mentioned previously, in order for VERA to be usable by industry, several of the industry partners have deployed smaller computer clusters (on the order of 500 processors) to test and apply VERA to real-world problems [5, 6].

CASL also has state-of-the-art collaboration facilities deployed to the various core partners to permit easy and frequent collaboration. These facilities include videoteleconferencing systems that integrate computer screen sharing, projection video monitors, and virtual whiteboards to any number of simultaneous participants. There is also a state-of-the-art Virtual Office Community and Computing (VOCC) Laboratory at ORNL that includes a system, the F-5 Storm, which is able to display scientific information in stereoscopic 3D with head- and gesture-tracking that allows a user to interact with and interrogate his or her data and calculation results in real-time (see Fig. 1).

Experimental validation of the calculations and modeling undertaken by CASL are provided at several levels. First, physics models are developed as a result of fundamental research. This research is typically undertaken in laboratory facilities by the academic and laboratory partners exploring specific phenomena such as material performance under operating reactor conditions and tabletop and/or largerscale T-H experiments. Individual components of VERA can then be validated against appropriate 


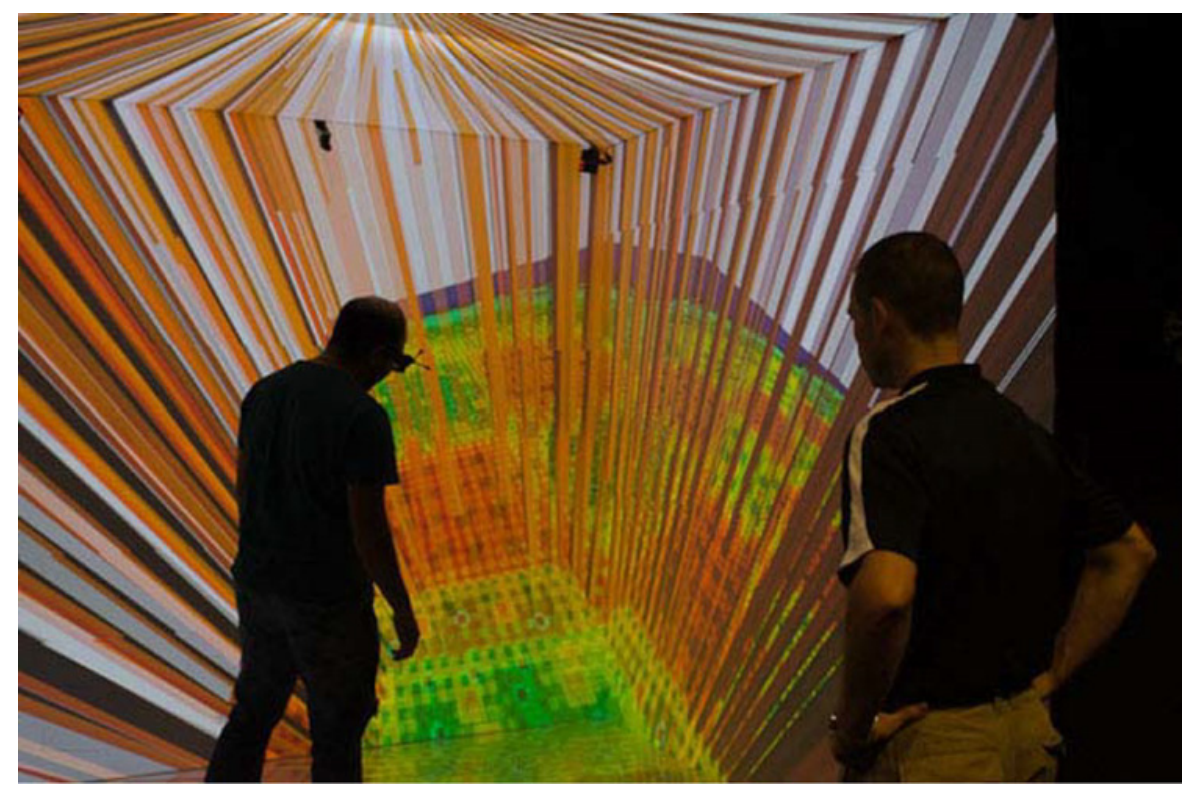

Figure 1. A User Interacting with a 3D Neutron Flux Distribution in the F-5 Storm System at ORNL.

datasets removing the effects of other phenomena to ensure that the physics for a particular module are behaving correctly. On another level, large scale validation takes place by comparing VERA calculations to operating or next-generation reactors. These comparisons are typically undertaken by the industry partners and use operational reactor data to validate the calculations.

\section{Progress to Date}

As a 5 year program, a roadmap exists that describes key capabilities that should be available each year in the following areas:

- Neutron Transport,

- Thermal Fluids with Conjugate Heat Transfer,

- Fuel \& Clad Performance,

- Coolant Chemistry,

- Structural Thermo-Mechanics,

- Physics Coupling, and

- Validation and Uncertainty Quantification.

Taking the area of Neutron Transport as an example, Year 1 capabilities require full-core 3D homogenous pin cell $S_{N}$ transport and full-core 2D/1D synthesis resolved pin cell MOC transport with $\mathrm{T}-\mathrm{H}$ coupling. Year 5 requires transient full-core $3 \mathrm{D}$ pin-resolved (either $\mathrm{S}_{\mathrm{N}}$ or MOC) transport with T-H coupling and 3D hybrid Monte Carlo with T-H coupling. Intervening years see a gradual transition in complexity between these two endpoints.

Furthermore, focus areas have goals that become progressively more challenging. For example, AMA progresses through 10 stages of analysis capabilities which include moving from 2D hot zero power (HZP) pin cell calculations to 3D HZP assembly calculations to physical reactor zero power physics tests and progressing to depletion and hot full power (HFP) operation. 


\section{$15^{\text {th }}$ ISRD}

In general, the roadmap and focus area timelines have been adhered to up to this point. Too numerous to list, it is recommended that the interested reader consult the R\&D - Publications area of the CASL website to understand the past and current state of the activities in each of these areas.

\section{Future Work \& Summary}

Given the structured organization and timeline for CASL at both the strategic and tactical levels, the future work consists primarily of continuing to execute the plan as stated. Continued success in this regard will provide the justification needed to extend CASL for an additional 5 years in order to continue improving the methodologies in the areas currently being pursued as well as to approach Challenge Problems in other areas in the next phase. There are already advocates for moving the improved modelling capabilities into the excore regions, both in- and ex-vessel, in order to use the transport methodologies to analyze in- and ex-vessel reactor dosimetry. This would be a boon to the reactor dosimetry community (by providing new tools and methods) and to CASL (to access an extensive collection of reactor benchmarks and measurement data).

In summary, CASL has shown good success in the $\sim 4$ years since its inception. Perhaps as important as the technical achievements is the strong collaboration between the laboratory, academic, and industry partners. This partnership is clearly a key contributor to the many technical successes and is a proven model for major technical endeavors in the future. It requires wide-ranging expertise from throughout a technical community to solve problems that face that community in a highly-structured way in order to produce tangible results.

The research and projects described herein were supported by the Consortium for Advanced Simulation of Light Water Reactors (http://www.casl.gov), an Energy Innovation Hub (http://www. energy.gov/hubs) for Modeling and Simulation of Nuclear Reactors under U.S. Department of Energy Contract No. DE-AC0500OR22725.

\section{References}

[1] Scale: A Comprehensive Modeling and Simulation Suite for Nuclear Safety Analysis and Design, ORNL/TM-2005/39, Version 6.1, June 2011. Available from Radiation Safety Information Computational Center at Oak Ridge National Laboratory as CCC-785

[2] T. M. Evans et al., Denovo: A New Three-Dimensional Parallel Discrete Ordinates Code in SCALE, Nuclear Technology, Vol. 171, No. 2, August 2010, pp. 171-200

[3] Consortium for the Advanced Simulation of Light Water Reactors Project Website, http://www.casl.gov

[4] TOP500 Supercomputer Sites Website, http://www. top500.org/, November 2013

[5] A. Godfrey et al., Operational Reactor Model Demonstration with VERA: Watts Bar Unit 1 Cycle 1 Zero Power Physics Tests, CASL Technical Report CASL-U-2013-0105-001, September 2013

[6] F. A. Franceschini et al., Westinghouse VERA Test Stand - Zero Power Physics Test Simulations for the AP1000 PWR, CASL Technical Report: CASL-U-2014-0012-001, March 2014

[7] CASL Project Summary Slides, CASL Viewgraph Collection: CASL-U-2011-0137-000, August 2011 